\title{
Evaluation of the use of systemic antimicrobial agents by professionals for the treatment of periodontal diseases
}

\author{
Aline Vicentini Monteiro', Fernanda Vieira Ribeiro', Renato Corrêa Viana Casarin', \\ Fabiano Ribeiro Cirano ${ }^{1}$, Suzana Peres Pimentel ${ }^{1}$, Márcio Zaffalon Casati ${ }^{1,2}$ \\ ${ }^{1}$ Area of Periodontics, School of Dentistry, Paulista University, São Paulo, SP, Brazil \\ ${ }^{2}$ Department of Prosthodontics and Periodontics, School of Dentistry of Piracicaba, University of Campinas (UNICAMP), Piracicaba, SP, Brazil
}

\begin{abstract}
Aim: To investigate the indication of systemic antimicrobial agents used by dental professionals for treatment of patients affected by periodontal diseases. Methods: Interviews by a questionnaire were held with 225 professionals of different dental specialties and who performed periodontal treatment. Results: Among interviewees, $94 \%$ indicated systemic antibiotics as a form of periodontal disease treatment. Their main indication was for periodontal abscesses $(80 \%)$ followed by aggressive periodontitis $(62 \%)$ and necrotizing diseases $(45 \%)$. The most frequently used antibiotics were amoxicillin $(81 \%)$ and metronidazole $(57 \%)$. The medications were indicated in association with mechanical therapy by $67 \%$ of the professionals. As regards the occasion of indication, $60 \%$ indicated systemic antibiotic therapy before and after mechanical periodontal scaling and root planing. Seventy-eight percent of the professionals indicated antibiotics associated with periodontal surgery for access to scaling, and $76 \%$ indicated it before and after the surgical procedure. Among the interviewees, $99 \%$ took into account systemic involvement for drug administration. Conclusions: It was concluded that a considerable portion of professionals indicate systemic antibiotic-therapy in an incoherent manner and in situations in which there is no indication for antibiotic use, or with ineffective protocols.
\end{abstract}

Keywords: periodontitis, anti-infective agents, questionnaires.

\section{Introduction}

Received for publication: August 26, 2013

Accepted: November 25, 2013

Correspondence to: Marcio Zaffalon Casati Departamento de Odontologia, Universidade Paulista (UNIP) Avenida Dr. Bacelar, 1212,

$4^{\circ}$ andar, CEP: 04026-002

Vila Clementino, São Paulo, SP, Brasil Phone/Fax: +55 1155864000 E-mail: mzcasati@gmail.com
Periodontal treatment is based on supra and subgingival dental biofilm control, performed by guidance on oral hygiene, professional supragingival biofilm removal and subgingival periodontal instrumentation. These therapeutic approaches alter the microbiota, allowing the establishment of a larger proportion of bacteria related to periodontal health. However, some studies demonstrated that mechanical therapy used alone, may not combat adequately some types of periodontopathogens, making it difficult to obtain or re-establish periodontal health ${ }^{1}$.

Studies demonstrated that the use of systemic antimicrobial agents adjunct to mechanical periodontal treatment may be an important therapeutic strategy in the treatment of chronic ${ }^{2-4}$ and aggressive ${ }^{5-9}$ periodontal diseases. These results were observed with the use of different antibiotics, such as amoxicillin/metronidazole ${ }^{4,8-11}$, azythromicin ${ }^{12-14}$, clindamycin ${ }^{15}$ and clarithromycin ${ }^{16}$, which showed favorable results as adjunct to therapy for aggressive periodontitis and chronic periodontal 
disease, when compared with the control group treated with mechanical therapy alone. In addition to the effects of systemic antibiotic therapy based on the isolated use of a certain drug, the use of associations of antibiotics has also shown promising clinical and microbiological results ${ }^{3,5,8,17}$.

Another factor of great importance for the correct indication of systemic antimicrobial agent use in therapy for periodontal alterations, in addition to the correct type of drug or association, is the occasion of using systemic antibiotic therapy. Studies indicated that due to the characteristics of dental biofilm, it is necessary to disorganize it mechanically before, or soon after the beginning of antimicrobial therapy, to optimize the medication ${ }^{18,19}$.

It is relevant to point out that the conscious use of systemic antibiotics is based on the possibility of adverse reactions to these medications, and particularly on the possibility of selecting resistant microorganisms ${ }^{20-22}$. Adverse reactions may vary from simple nausea and headache, up to the presence of pseudomembranous colitis which, if not correctly treated, may result in the death of the patient affected by this infection. In addition, bacterial resistance is the most important negative factor in the use of indiscriminate and incorrect systemic antibiotic protocols ${ }^{20}$.

In 1996, was made an evaluation of systemic antimicrobial agents use by professionals for periodontal treatment ${ }^{23}$. In this study, was demonstrated the abusive use of systemic antimicrobial agents. Among dentists who performed periodontal treatment, $85 \%$ of them mentioned the use of systemic antibiotics in the treatment of chronic periodontitis, without considering the severity of the disease. Furthermore, it was observed that the majority of the interviewed professionals prescribed antibiotics for patients with acute processes (periodontal abscesses and necrotizing diseases) without consideration of the patient's systemic conditions.

In view of past evidence of abusive use of antibiotic therapy by professionals who perform therapeutic periodontal procedures, and knowing about the risks of indiscriminate use of this type of medication, the aim of the present study was to make an up-to-date evaluation of systemic antimicrobial agents indication by dental professionals who perform periodontal therapy.

\section{Material and methods}

\section{Study Design}

This study had a cross-sectional, observational design, using structured questionnaires with multiple-choice questions that allowed completion of responses.

\section{Sample}

In the present study, 225 dentists who performed periodontal treatment in private clinics or in public service voluntarily participated in the study. Professionals involved in teaching activities in dental schools were excluded from the survey. All the professionals were interviewed by the same person, who followed the structured questionnaire adapted for this study. Before the study interviews were held, the questionnaire was tested as regards aspects of understanding the questions, interviewing five dentists not involved in this project. Before the interview, the participants were informed about the research and signed a form of free and informed consent. All the procedures performed were approved by the Ethics Commission of the Paulista University (Protocol No. 539/10 CEP/ICS/UNIP).

Nine multiple choice questions were asked with regards the use of systemic antibiotic therapy in the treatment of periodontal diseases. During initial questioning, the professionals reported whether they used or not antibiotics in the treatment of periodontal diseases. Subsequently, they answered questions about the types of periodontal diseases in which antibiotic therapy was indicated, and could choose one or more of the following clinical situations: gingivitis, chronic periodontitis, aggressive periodontitis, periodontitis modified by systemic factors, periodontal abscesses, and necrotizing or other diseases. Questions were also asked as regards the disease severity in which the medications should be indicated (slight, moderate or advanced).

After this, the professionals were asked whether the patient's systemic condition was taken into consideration for prescription of antibiotics. Next, the professionals answered questions about whether they indicated the use of antibiotics in association with mechanical therapy, or whether they prescribed medication as monotherapy, and about the occasion of using the medication (prescribing the medication before, before and after, or only after the root scaling and planing procedures). In addition, the professionals were asked whether they prescribed or not antibiotics after performing periodontal surgery for access to root scaling. In cases in which they indicated the use of antibiotics combined with periodontal surgery, the professionals were asked about the occasion of medication use (indicating medication before, before and after, or only after the surgical procedures).

The last question asked was about the used medication (Amoxicillin, Tetracycline, Metronidazole, Ciprofloxacin, Clindamycin, Azithromycin, associations of medications and others).

\section{Results}

The replies to each question were computed in terms of relative frequency and as a whole, they were analyzed by means of descriptive statistics. Interviews were held with 225 dentists from different specialties (Figure 1). Although the professionals were from different specialties (public health $2.3 \%$, pediatric dentistry $3.8 \%$, restorative dentistry $6.1 \%$, oral surgery $9.9 \%$, endodontics $12.4 \%$, orthodontics $12.4 \%$, periodontics $14.6 \%$, prosthesis $15.3 \%$ and implantology $23.2 \%$ ), they all performed periodontal therapy on their patients.

As regards the first question about whether they indicated or not antibiotic therapy in the treatment of periodontitis, only $6 \%$ of the professionals reported that they did not use systemic antibiotics in the treatment of periodontal diseases. The professionals who used antibiotic therapy reported using 

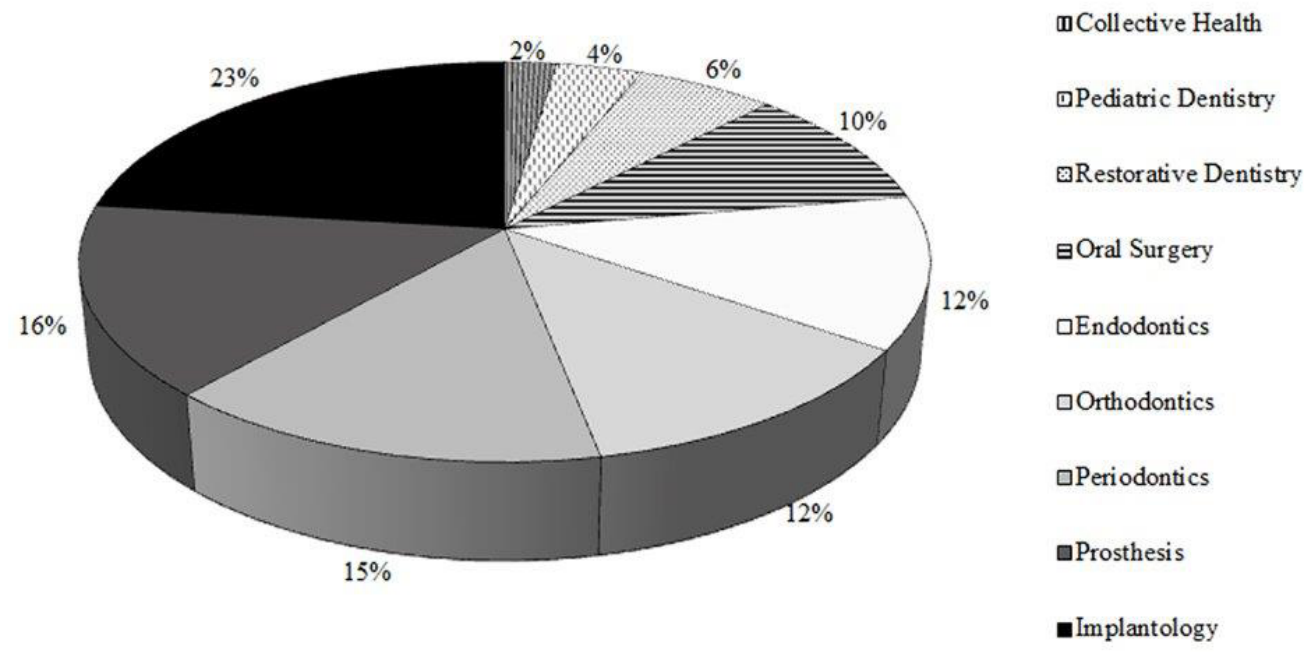

Fig. 1 - Frequency of indication of systemic antimicrobials in the treatment of periodontal diseases by dentists from different specialties.

them mainly in cases of periodontal abscesses $(80 \%)$, aggressive periodontitis $(62 \%)$ and necrotizing diseases $(45 \%)$ (Figure 2).

It should be pointed out that some of the professionals indicated more than one clinical situation for the use of systemic antibiotic therapy. As regards the disease severity for which the medications should be indicated, $87 \%$ of the professionals indicated antibiotic therapy for advanced periodontitis, $38 \%$ for moderate and $6 \%$ for slight periodontitis.

Asked whether they took the systemic involvement of the patient into consideration when opting to use antibiotic therapy, $99 \%$ of the dentists reported that they did take this into consideration when prescribing antibiotics, while $1 \%$ did not.

When asked about the use of antibiotics as adjunct to mechanical therapy, it was observed that most of the interviewed professionals used antibiotic therapy in association with root instrumentation (67\%), although a considerable portion $(33 \%)$ indicated it without combination with mechanical therapy. When asked about the occasion of using antibiotics, in case of association with the root scaling and planing, most of the professionals $(60 \%)$ reported they indicated the use of medication both before and after nonsurgical mechanical instrumentation (i.e. scaling and root planing) (Figure 3).

Asked about the prescription of antibiotics after performing periodontal surgery for access to root scaling, $78 \%$ of the professionals replied they indicated the use of medication associated with surgeries, while $22 \%$ did not prescribe antibiotics associated with surgeries. As regards the occasion of using antibiotics in cases of periodontal surgeries, most of the professionals $(76 \%)$ reported that they indicated the use of medication both before and after the surgical procedure (Figure 4).

With regard to the choice of medications, a predominance of bactericide antibiotic prescriptions (amoxicillin and metronidazole) was observed (Figure 5).

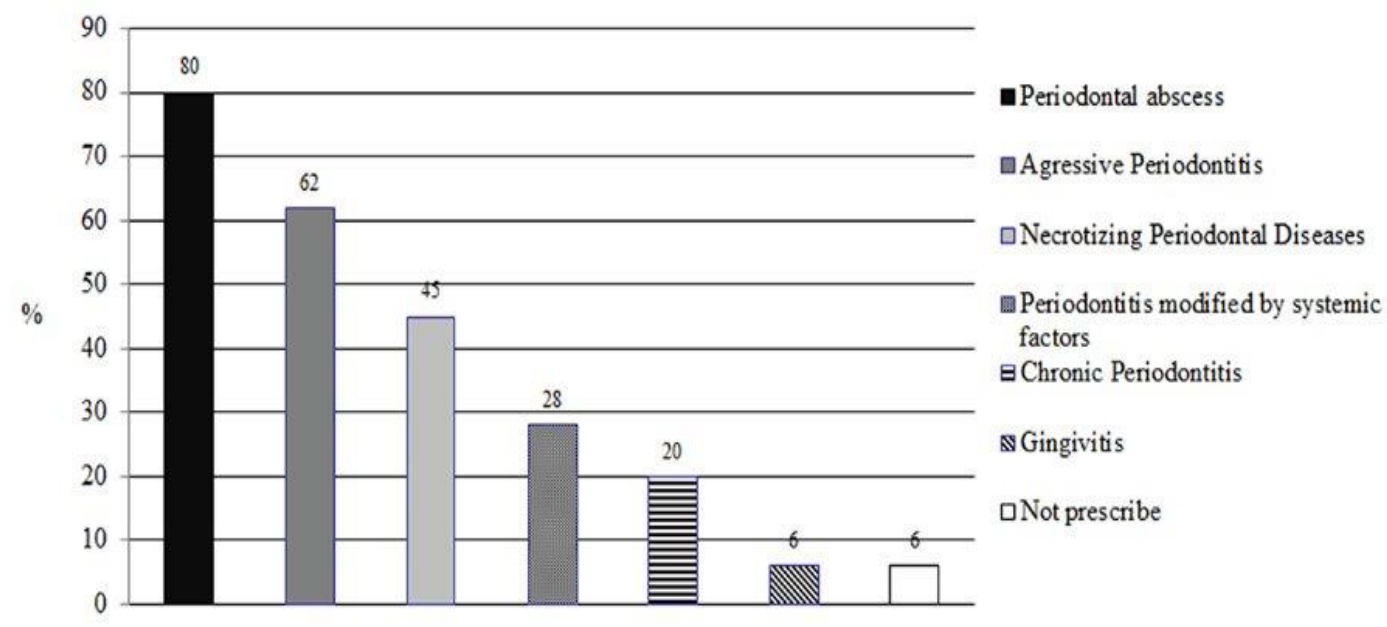

Fig. 2 - Number and percentage of indications for the use of antimicrobials in the different types of periodontal disease. 


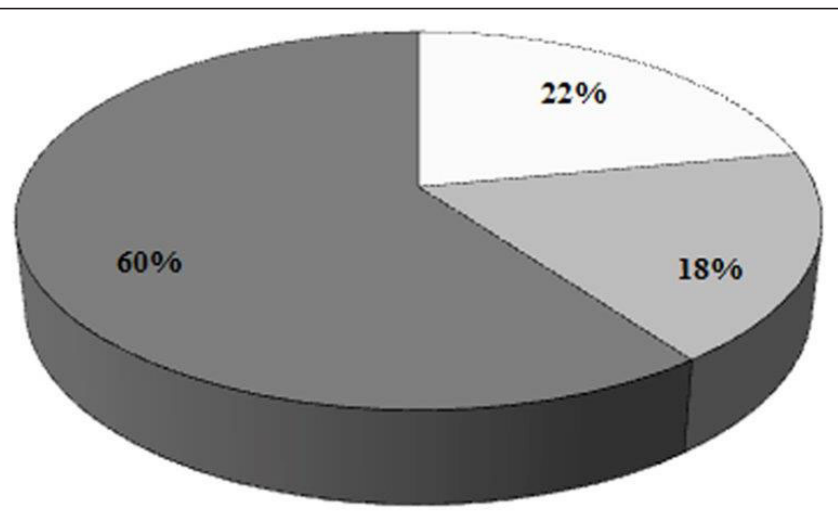

\section{$\square$ Before SRP $\quad \square$ After SRP $\square$ Before and after SRP}

Fig. 3 - Frequency of indication of the use of antimicrobials according to the occasion of administration, in the case of association with scaling and root planing.

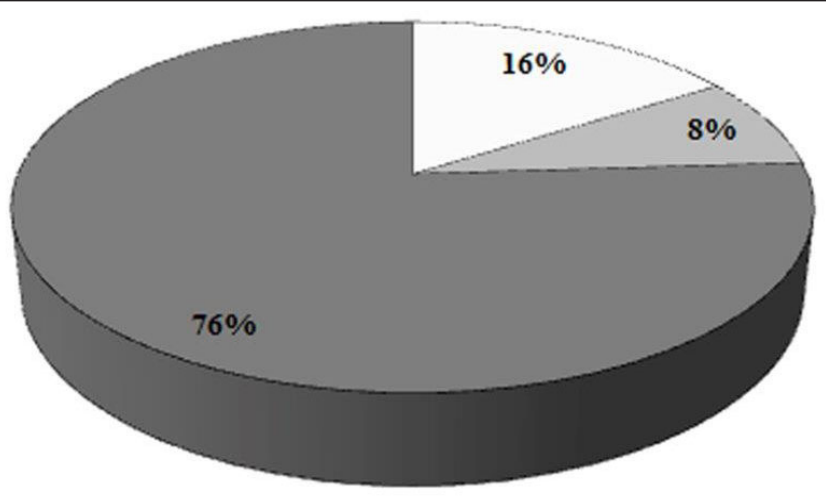

$\square$ Before surgery $\square$ After surgery $\square$ Before and after surgery

Fig. 4 - Frequency of indication of the use of antimicrobials according to the occasion of administration in the case of association with periodontal surgeries.

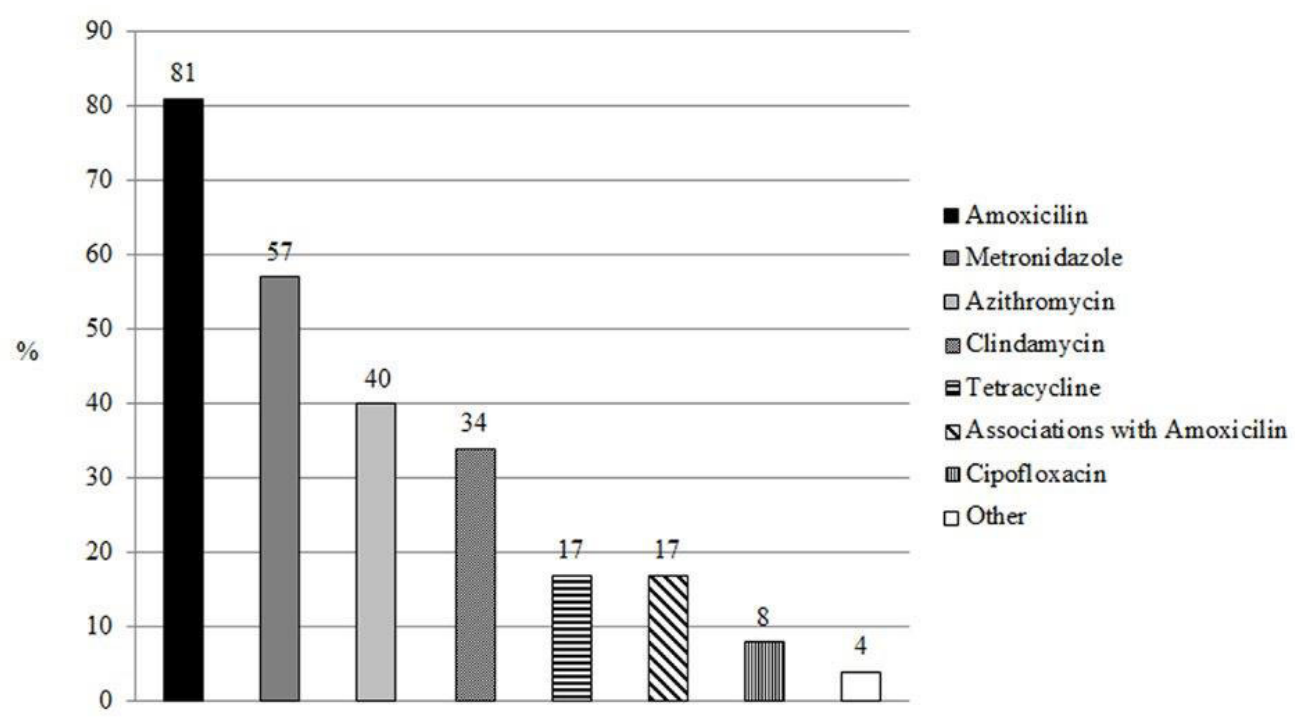

Fig. 5 - Number and percentage of the medications indicated by the interviewed professionals. 


\section{Discussion}

In view of previous evidence that demonstrated abusive use of antibiotics by professionals who perform therapeutic periodontal procedures, and bearing in mind the risks associated with the indiscriminate use of this type of medication, the aim of this study was to make an up-to-date evaluation of the indication of systemic antimicrobial agents by dental professionals who perform periodontal therapy. According to the findings of the present study, a large portion of the interviewees (92\%) was shown to use antibiotics in the treatment of periodontal diseases, and that in $80 \%$ of the cases, this indication was being directed to the treatment of periodontal abscesses.

Different therapies have been proposed for the treatment of periodontal abscesses, which turned the subject controversial ${ }^{1,24,25}$. However, the use of antibiotics must be restricted to individuals with systemic involvement such as bad feeling, fever, prostration and lymphoadenopathy ${ }^{26}$. Indeed, in the present study most of the interviewed professionals (99\%) considered this aspect when taking the decision to prescribe the medication. However, as only a small portion of periodontal abscesses develop to a systemic involvement, and $80 \%$ of the dentists prescribe antibiotics for the treatment of this acute process, this may indicate excessive use of such medication for this purpose.

In the present study, a considerable portion of the interviewees $(62 \%)$ also reported the use of systemic antibiotic therapy for the treatment of aggressive periodontitis. In this regard, various researchers showed that there was a significant benefit of their use as adjunct to mechanical treatment, when compared with individuals who received mechanical treatment only ${ }^{5,6}$. Haas et al. ${ }^{12}$ (2008) used an antibiotic regime of $500 \mathrm{mg}$ azythromicin, once a day, for 3 days, associated with root scaling and planing, and after 12 months of follow-up, obtained significant improvement in the evaluated clinical parameters (probing depth and clinical attachment level) in comparison with the group that received mechanical therapy alone. In addition to the clinical benefits, some recent studies also reported the positive effects of the adjunct use of antibiotics on the subgingival microbiota of individuals with aggressive periodontitis. In a randomized, double-blind, placebocontrolled study, 30 individuals with aggressive periodontitis received root scaling and planing alone, or combined with $400 \mathrm{mg}$ of metronidazole plus $500 \mathrm{mg}$ of amoxicillin, 3 times a day for 14 days $^{5}$. The individuals who received the combination of antibiotics showed better results in the microbial profile, presenting smaller proportions of the red and orange complexes after treatment ${ }^{5}$. Rodrigues et al. ${ }^{7}$ (2012) also observed a significant reduction in the quantity of subgingival Tannerella forsythensis (T.f.) and Treponema denticola (T.d.) in patients with aggressive periodontitis treated with amoxicillin and metronidazole, in association with mechanical treatment. Although there is scientific evidence supporting the use of systemic antibiotics adjunct to root instrumentation in the treatment of aggressive periodontitis, care must be taken when diagnosing cases of aggressive periodontitis, and differentiate them from advanced chronic periodontitis, for example, since there is a set of specific characteristics for the diagnosis of this type of periodontal disease. This caution is relevant, considering that the use of antibiotics in other types of periodontal disease might not be a suitable option.

The results of the present study also demonstrated that a great number of professionals (45\%) indicate the use of antibiotics in cases of necrotizing periodontitis. Some studies ${ }^{27}$ affirmed that systemic antibiotics are generally not necessary in the treatment of necrotizing processes, provided that mechanical therapy of the site is sufficient to efficiently contain the disease progression. Thus, only in the few cases in which the patient's response to mechanical therapy is minimal, or in cases in which the patient presents systemic involvement, is indicated the supplementary use of antibiotics. Bearing in mind that in the present study, the use of antibiotics was prescribed by almost half of the professionals in the treatment of this periodontal condition, it suggests once again that professionals are making excessive use of this medication.

As regards the adjunct use of antibiotics in the treatment of chronic periodontitis, it has been mentioned that generally an efficient clinical response may be obtained by performing mechanical therapy only ${ }^{13}$. Studies emphasized that in order to provide maximum benefit, there is need to reserve the use of these antibiotic agents for individuals with moderate or severe diseases ${ }^{18,28}$. Furthermore, when mechanical therapy alone does not stop the process or prevent recurrence of the disease, a situation more prevalent in smokers, the adjunct use of antibiotics would be justified ${ }^{2,29}$. Some authors ${ }^{30}$ demonstrated that the use of Azithromycin, in combination with root scaling and planing improved the results of nonsurgical therapy in smokers. According to the authors, greater reduction in probing depth and gains in clinical attachment levels in patients with moderate to advanced attachment loss have been observed when compared with patients who received root scaling and planing only. Clinical and microbiological benefits have also been obtained by some authors $^{2}$ with the combination of amoxicillin and metronidazole adjunct to mechanical therapy in the treatment of chronic periodontitis in smokers. In the present study, $20 \%$ of the professionals reported indicating the use of antibiotics in the treatment of chronic periodontitis, irrespective of the severity of the disease or the patient's smoking habit, aspects considered relevant when the use of antibiotic therapy is referred to in chronic periodontal disease therapy. In spite of these studies demonstrating positive results with the use of antibiotics as adjunct to the treatment of chronic periodontitis ${ }^{2,3,8,18,28,30}$, a recent systematic review showed that there is still insufficient and inconclusive evidence to support the use of systemic antibiotics ${ }^{31}$.

Although most of the mentioned professionals concern about associating antibiotic with mechanical therapy, 33\% of the professionals used antibiotic therapy alone. According to previous evidence, antibiotics must not be used as 
substitutes for mechanical instrumentation of the site ${ }^{18,32}$ whether it is performed surgically or not, due to the fact that biofilm is relatively resistant to antimicrobial agents ${ }^{19}$ unless it is mechanically disorganized.

The literature is full of studies demonstrating the effectiveness of antibiotics associated with instrumentation, but there is no precise definition of the best occasion to prescribe them. In the present study, most of the interviewed professionals $(60 \%)$, mentioned using antibiotics before and after non-surgical mechanical instrumentation. It was shown that the administration of antibiotic therapy during the mechanical therapy, that is to say, not before and not after instrumentation, provides greater reduction in probing depth and greater attachment gain in deep pockets ${ }^{32}$. Therefore, it seems that the best form of using systemic antibiotic therapy would be in association with mechanical treatment of all the regions with periodontal involvement, so that the period of antibiotic administration covers the time required to perform full-mouth mechanical therapy, preferably less than one week.

As regards the use of antimicrobial agents in the periodontal surgical procedures of root scaling and planing, it was found that the majority of the professionals (76\%), used antibiotics before and after the procedures. It is important to point out that although some authors have suggested that the adjunct use of antibiotics may reduce pain and swelling and improve healing, there is no sufficient evidence to support their use associated with periodontal surgery ${ }^{32}$. In an extensive review ${ }^{33}$ of all the records available of periodontal surgeries in a sample of 395 patients and 1.053 periodontal surgery procedures, it was demonstrated that there were no benefits from antibiotics indication in these cases, due to the low rate of post-operative infections. Therefore, it is probable that the large number of indications found in the present study were made empirically. One of the few situations in which the use of systemic antibiotics before surgical procedures could be indicated, is for the prevention of bacterial endocarditis ${ }^{34}$.

As discussed in this study, although certain periodontal conditions have been benefitted by the adjunct use of antibiotics, there is no protocol or consensus as regards to medications that must be used in each case ${ }^{1}$. Some studies showed that the association of amoxicillin (a penicillin with broader spectrum) and metronidazole (a nitroimidazole compound) - especially in a regime of $400 \mathrm{mg}$ of metronidazole plus $500 \mathrm{mg}$ of amoxicillin, 3 times a day for 7-14 days - plays an important role in treating anaeroberelated infection in the oral cavity and may significantly improve the short-term results of non-surgical therapy in patients with aggressive periodontitis ${ }^{5,6,9,10}$, diminishing the need for surgical therapy. This association has shown good results by presenting a synergic effect between the drugs ${ }^{9,11,35}$. Azythromicin, a member of a new subclass of macrolide, the azalides, has also been shown to be effective as adjunct to the treatment of aggressive periodontitis (regime of $500 \mathrm{mg}$ de azythromicin, once a day, for 3 days $)^{12}$. Therefore, one may affirm that there is scientific support for the use of the antibiotics most frequently indicated by the professionals in the present study (amoxicillin, metronidazole and azythromicin), for the treatment of periodontal diseases, mainly as adjunct to mechanical therapy in the management of aggressive periodontitis.

An important subject refeents to the use of antibiotic therapy is the bacterial resistance, which is associated with excessive and/or indiscriminate use of antibiotics ${ }^{21,22}$. Ardila et al. ${ }^{22}$ (2010) revealed that periodontal microorganisms in patients with chronic periodontitis can be resistant to the antimicrobial agents frequently used in anti-infective periodontal therapy, such as amoxicillin and metronidazole, supporting the position that the indiscriminate use of antimicrobials leads to the appearance of more highly antibiotic-resistant strains of bacteria associated with periodontal diseases. In the survey conducted 15 years ago on the same subject as in of the present study ${ }^{23}$, only $7 \%$ of the professionals reported taking into consideration the systemic manifestation of infection when indicating antibiotics, whereas in the present study $99 \%$ reported considering this involvement when indicating this type of medication. This result demonstrates that there has been a considerable change in the knowledge of professionals who indicate antibiotic therapy nowadays. Nevertheless, although professionals reported being more prepared for, and conscious of indicating antibiotic therapy in most indications, it could still be observed that there was indiscriminate use, which was scarcely based on scientific evidence.

An important aspect to be highlighted is related to the type of questionnaire used in the present study. Although a structured multiple-choice questionnaire was used during the interviews, alternative options of answers or other types of questions could have led to different outcomes.

In view of the results obtained in the present study, it may be concluded that systemic antibiotics are still used by dentists in an incoherent manner, and scarcely based on the scientific advancements published in the literature, for clinical situations in which antibiotics are not indicated or using protocols that are not effective in periodontal therapy.

\section{References}

1. Herrera D, Matesanz P, Bascones-Martínez A, Sanz M. Local and systemic antimicrobial therapy in periodontics. J Evid Based Dent Pract. 2012; 12: 50-60.

2. Matarazzo F, Figueiredo LC, Cruz SE, Faveri M, Feres M. Clinical and microbiological benefits of systemic metronidazole and amoxicillin in the treatment of smokers with chronic periodontitis: a randomized placebo controlled study. J Clin Periodontol. 2008; 35: 885-96.

3. Cionca N, Giannopoulou C, Ugolotti G, Mombelli. Amoxicillin and metronidazole as an adjunct to full-mouth scaling and root planing of chronic periodontitis. J Periodontol. 2009; 80: 364-71.

4. Sgolastra F, Gatto R, Petrucci A, Monaco A. Effectiveness of systemic amoxicillin/metronidazole as adjunctive therapy to scaling and root planing in the treatment of chronic periodontitis: a systematic review and metaanalysis. J Periodontol. 2012; 83: 1257-69.

5. Mestnik MJ, Feres M, Figueiredo LC, Duarte PM, Lira EAG, Faveri M. Short-term benefits of the adjunctive use of metronidazole plus amoxicillin in the microbial profile and in clinical parameters of subjects with generalized aggressive periodontitis. J Clin Periodontol. 2010; 37: 353-65. 
6. Yek EC, Citan S, Topcuoglu N, Kulekci G, Issever H, Kantarci A. Efficacy of amoxicillin and metronidazole combination for the management of generalized aggressive periodontitis. J Periodontol. 2010; 81: 964-74.

7. Rodrigues AS, Louren ODS, Lima Neto LG, Pannuti CM, Crespo Hirata $\mathrm{RD}$, Hirata $\mathrm{MH}$, et al. Clinical and microbiological evaluation, by real-time $\mathrm{pcr}$, of non-surgical treatment of aggressive periodontitis associated with amoxicillin and metronidazole. J Periodontol. 2012; 83: 744-52.

8. Silva MP, Feres M, Sirotto TA, Soares GM, Mendes JA, Faveri M, et al. Clinical and microbiological benefits of metronidazole alone or with amoxicillin as adjuncts in the treatment of chronic periodontitis: a randomized placebo-controlled clinical trial. J Clin Periodontol. 2011; 38: 828-37.

9. Arweiler NB, Pietruska M, Skurska A, Doliñska E, Pietruski JK, Bläs M, et al. Nonsurgical treatment of aggressive periodontitis with photodynamic therapy or systemic antibiotics. Three-month results of a randomized, prospective, controlled clinical study. Schweiz Monatsschr Zahnmed. 2013; 123: $532-44$

10. Silva-Senem MX, Heller D, Varela VM, Torres MC, Feres-Filho EJ, Colombo AP. Clinical and microbiological effects of systemic antimicrobials combined to an anti-infective mechanical debridement for the management of aggressive periodontitis: a 12-month randomized controlled trial. J Clin Periodontol. 2013; 40: 242-51.

11. Zandbergen D, Slot DE, Cobb CM, Van der Weijden FA. The clinical effect of scaling and root planing and the concomitant administration of systemic amoxicillin and metronidazole: a systematic review. J Periodontol. 2013; 84: 332-51.

12. Haas AN, De Castro GD, Moreno T, Susin C, Albandar JM, Oppermann $\mathrm{RV}$, etal. Azithromycin as an adjunctive treatment of aggressive periodontitis: 12-months randomized clinical trial. J Clin Periodontol. 2008; 35: 696-704.

13. Sampaio E, Rocha M, Figueiredo LC, Faveri M, Duarte PM, Gomes Lira $E A$, et al. Clinical and microbiological effects of azithromycin in the treatment of generalized chronic periodontitis: a randomized placebo-controlled clinical trial. J Clin Periodontol. 2011; 38: 838-46.

14. Han B, Emingil G, Ozdemir G, Tervahartiala T, Vural C, Atilla G, et al. Azithromycin as an adjunctive treatment of generalized severe chronic periodontitis: clinical, microbiological and biochemical parameters. J Periodontol. 2012; 83: 1480-91.

15. Sigusch B, Beier M, Klinger G, Pfister W, Glockmann E. A 2-step nonsurgical procedure and systemic antibiotics in the treatment of rapidly progressive periodontitis. J Periodontol. 2001; 72: 275-83.

16. Pradeep AR, Kathariya R. Clarithromycin, as an adjunct to non-surgical periodontal therapy for chronic periodontitis: A double blinded, placebo controlled, randomized clinical trial. Arch Oral Biol. 2011; 56: 1112-9.

17. Cionca N, Giannopoulou C, Ugolotti G, Mombelli A. Microbiologic testing and outcomes of full-mouth scaling and root planing with or without amoxicillin/ metronidazole in chronic periodontitis. J Periodontol. 2010; 81: 15-23.

18. Colombo AP, Teles RP, Torres MC, Rosalem W, Mendes MC, Souto $\mathrm{RM}$, et al. Effects of non-surgical mechanical therapy on the subgingival microbiota of Brazilians with untreated chronic periodontitis: 9-month results. J Periodontol. 2005; 76: 778-84.

19. Marsh PD. Dental plaque: biological significance of a biofilm and community lifestyle. J Clin Periodontol. 2005; 32: 7-15.

20. Feres M, Haffajee AD, Allard K, Som S, Goodson S, Socransky SS. Antibiotic resistance of subgingival species during and after antibiotic therapy. J Clin Periodontol. 2002; 29: 724-35.

21. Goossens, H. Causal link confirmed between antibiotic use and resistance. Lancet. 2007; 369: 482-90.

22. Ardila CM, Granada MI, Guzmán IC. Antibiotic resistance of subgingival species in chronic periodontitis patients. J Periodontal Res. 2010; 45: 557-63.

23. Casati MZ, Nociti Junior FH, Sallum EA, Toledo S, Sallum AW. Evaluation of using antimicrobial agents in the therapy of periodontal diseases. Periodontics. 1996; 5: 335-40.

24. Herrera D, Roldan S, Sanz M. The periodontal abscess: a review. J Clin Periodontol. 2000; 27: 377-86.

25. Lypka M, Hammoudeh J. Dentoalveolar infections. Oral Maxillofac Surg Clin North Am. 2011; 23: 415-24.

26. Herrera D, Roldan S, O'Connor A, Sanz M: The periodontal abscess (II). Short term clinical and microbiological efficacy of 2 systemic antibiotic regimes. J ClinPeriodontol. 2000; 27: 395-404.
27. Bermejo-Fenoll A, Sánchez-Pérez A. Necrotising periodontal diseases. Med Oral Patol Oral Cir Bucal 2004; 9(Suppl): 114-9; 108-14.

28. Haffajee A.D, Torresyap G, Socransky SS. Clinical changes following four different periodontal therapies for the treatment of chronic periodontitis: 1 year results. J Clin Periodontol. 2007; 34: 243-53.

29. Albandar JM. Adjunctive antibiotics with nonsurgical periodontal therapy improve the clinical outcome of chronic periodontitis in current smokers. J Evid Based Dent Pract. 2012; 12: 63-6.

30. Mascarenhas P, Gapski R, Al-Shammari K, Hill R, Soehren S, Fenno $\mathrm{JC}$, et al. Clinical response of azithromycin as an adjunct to non-surgical periodontal therapy in smokers. J Periodontol. 2005; 76: 426-36.

31. Angaji M, Gelskey S, Nogueira-Filho G, Brothwell D. A systematic review of clinical efficacy of adjunctive antibiotics in the treatment of smokers with periodontitis. J Periodontol. 2010; 81: 1518-28.

32. Herrera D, Alonso B, Leon R, Roldan S, Sanz M. Antimicrobial therapy in periodontitis: the use of systemic antimicrobials against the subgingival biofilm. J Clin Periodontol. 2008; 35: 45-66.

33. Powell CA, Mealey BI, Deas D, McDonnell HT, Mortiz AJ. Post-surgical infections: prevalence associated with various periodontal surgical procedures. J Periodontol. 2005; 76: 329-33.

34. Wilson W, Taubert KA, Gewitz M, Lockhart PB, Baddour LM, Levison M, et al. Prevention of infective endocarditis: guidelines from the American Heart Association: a guideline from the American Heart Association Rheumatic Fever, Endocarditis and Kawasaki Disease Committee, Council on Cardiovascular Disease in the Young, and the Council on Clinical Cardiology, Council on Cardiovascular Surgery and Anesthesia, and the Quality of Care and Outcomes Research Interdisciplinary Working Group. J Am Dent Assoc 2007; 138: 739-45,747-60.

35. Slots J. Low-cost periodontal therapy. Periodontol. 2000.2012; 60: 110-37. 